\title{
Generation and Dynamics Analysis of N-Scroll Existence via Four Different Functions in Translation Chaotic System
}

\author{
Yue Liu* \\ College of Electrical and Electronic Engineering, Changchun University of Technology, Changchun 130012, China \\ ${ }^{*}$ Corresponding author
}

\begin{abstract}
In the three-dimensional ordinary differential equations, a systematic methodology for generating and designing $\mathrm{N}$-scroll chaotic attractors have been proposed by Liu in 2016, which was named the translation chaotic system and the translation transformation principle, respectively. In this paper, generation $\mathrm{N}$-scroll existence four different functions are been proposed. They are absolute function, sign function, the piecewise and hyperbolic function. Then, the dynamics properties in the chaotic system via different methods are investigated in detail. Furthermore, MATLAB simulation results show that these methods emerge similar but topologically non-equivalent chaotic attractors.
\end{abstract}

Keywords-translation chaotic system; dynamics properties; lyapunov exponent; hopf bifurcation; poincaré map

\section{INTRODUCTION}

Chaos theory, with relativity and quantum mechanics, is called one of the greatest discoveries of the twentieth Century. Earlier, scientists had realized that some deterministic dynamical systems involving only a few variables could exhibit complexity reminiscent of many-particle systems if the dynamics is chaotic, as can be quantified by the existence of a positive Lyapunov exponent, Hopf bifurcation and Poincaré map[1-5]. More, topological mixing[6] and periodicity[7] are also important factors from popular accounts of chaos, which equate chaos with sensitivity to initial conditions. Over the past six decades, chaos theories and chaos applications continue to be a very active area of research, involving many different disciplines[6, 8-17] (mathematics, topology, physics, etc.). In 1963, Lorenz found the Lorenz system was marked as the first remarkable chaotic model, which has just been mathematically confirmed to exist[8]. Since then, many scientists have begun to investigate the $N$-scroll chaotic attractors. Many research results have been obtained, such as generalized Lorenz canonical form[18,19], multi-wing butterfly attractors[20], Rössler systems[21] and so on. It is worth noting that these new chaotic systems are three dimensional quadratic autonomous ODEs. Subsequently, Chua circuit[22.23], created by Chua L.O. in 1983, and the other new $N$-scroll chaotic attractors[24-28] are obtained. They are three dimensional autonomous ODEs.

Inspired by previous works, the above research results are summarized. The translation-type chaotic systems were proposed $^{[29,30]}$ by Liu in 2016. This new type of chaotic system is composed of three subsystems, and one is named the translation chaotic system $\left(a_{12} a_{21}=0\right)$. Also, based on the translation transformation principle, the $N$-scroll attractors are obtained by the successful design criterion. In this paper, we propose the other four different design functions, which can generation $N$-scroll existence in the translation chaotic system. They are absolute function, sign function, the piecewise and hyperbolic function. Then, the dynamics properties are investigated in detail. Furthermore, MATLAB simulation results show that these chaotic attractors are similar but topologically non-equivalent. Undoubtedly these results will improve this chaotic system application value in the future.

We now outline the remainder of this paper. The translation chaotic system and translation transformation principle are given in Section II. In Section III, four different design functions are proposed, which can generation $N$-scroll existence. Then, simulation and the dynamics behaviors are also given to illustrate. Finally, concluding remarks are given in Section IV.

\section{TheORETICAL Design OF N-SCROll ChaOtiC ATTRACTORS}

The translation-type chaotic system is defined by the following ODEs:

$$
\left[\begin{array}{l}
\frac{d x_{1}}{d t} \\
\frac{d x_{2}}{d t} \\
\frac{d x_{3}}{d t}
\end{array}\right]=\left[\begin{array}{lll}
a_{11} & a_{12} & a_{13} \\
a_{21} & a_{22} & a_{23} \\
a_{31} & a_{32} & a_{33}
\end{array}\right] \cdot\left[\begin{array}{c}
X_{1} \\
X_{2} \\
x_{3}
\end{array}\right]+F(X)=A X+F(X)
$$

where $\mathrm{x}_{1}, \mathrm{x}_{2}, \mathrm{x}_{3}$ are state variables, $a_{i j}(1 \leq i \leq 3,1 \leq j \leq 3)$ are real parameters and $F(X)$ is the design criterion for constructing $2 N+1$-and 2(N+1)-scrolls based on the translation transformation principle[30]. By setting the some parameters and translation criterion, we can get three different subsystems.

\section{A. Translation Chaotic System}

The system (1), let $x_{1}=x, x_{2}=y, x_{3}=z, a_{21}=a_{22}=0, a_{23}=b$. Here, we would obtain the following simplest possible candidate system, which satisfies the condition $a_{12} a_{21}=0$ : 


$$
\left\{\begin{array}{l}
\frac{d x}{d t}=a_{11} x+a_{12} y+a_{13} z \\
\frac{d y}{d t}=b z \\
\frac{d z}{d t}=a_{31} x+a_{32} y+a_{33} z
\end{array}\right.
$$

where $x, y, z$ are state variables.

The system (2) gives us some basic information. Firstly, the system is a nonlinear ODEs, but it is unstable, dissipative and easily falling into the chaotic regime, and the scroll trajectory is bounded. Secondly, the system has equilibrium points, one of them in the origin $O(0,0,0)$. By the RouthHurwitz criterion, and the dissipation, the following conditions are satisfied: $a_{11}<-a_{33}$.

\section{B. Translation Transformation Principle}

In order to obtain $2(N+1)$ - and $2 N+1$-scroll attractors, we describe the new translation transformation principle as shown in Figure 1.

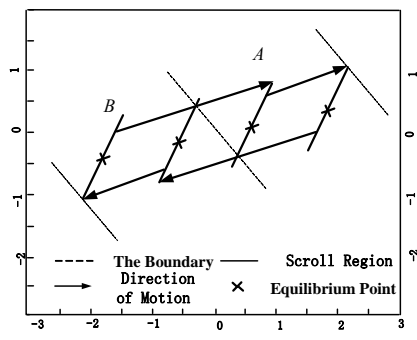

(a)

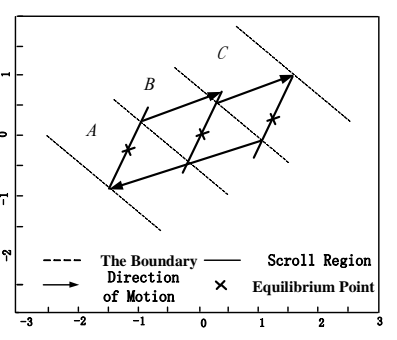

(b)
FIGURE I. TRANSLATION TRANSFORMATION PRINCIPLE: (a)4SCROLL; (b) 3-SCROLL

With enough time and the specific parameterization, the combination of all motion trajectories forms. It obtained $2(N+1)$ - and $2 N+1$-scroll attractors $(N=1,2,3,4, \ldots)$.

\section{GENERATION AND DYNAMIC BEHAVIORS OF N-SCROLL VIA FOUR DIFFERENT FUNCTIONS}

\section{A. Higher Number of Scrolls via Absolute Function}

To unified variables $(x, y, z)$, the dimensionless state equation of system (2) model is described by, let $a_{11}=-a, a_{12}=$ $0, a_{13}=r, a_{31}=0, a_{32}=-b, a_{33}=c$.

$$
\left\{\begin{array}{l}
\frac{d x}{d t}=-a[x-f(x+y+z)]+r[z-f(x+y+z)] \\
\frac{d y}{d t}=b[z-f(x+y+z)] \\
\frac{d z}{d t}=-b[y-f(x+y+z)]+c[z-f(x+y+z)]
\end{array}\right.
$$

where $x, y, z$ are state variables, and $a, b, c, r$ are real parameter. And $f(x+y+z)$ is the nonlinear absolute function, which is the translational transformation criterion for constructing scroll, as below:

$$
f(x+y+z)=A \times(|x+y+z+2 A|-|x+y+z-2 A|)
$$

When $a=4, r=2.4, b=4.4, c=0.2$ the system(3) can display single and double-scroll attractors simultaneously, as shown in Figure 2. And real $A(A \geq 0)$ is related to the position of the equilibrium point, at $( \pm 2 A, \pm 2 A, \pm 2 A)$.

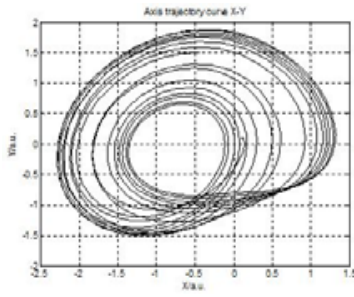

(a)

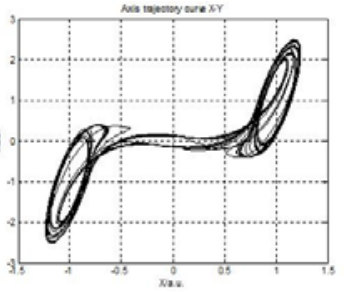

(b)
FIGURE II. SCROLL ATTRACTORS BY THE STATE QRUATION:

$$
\text { (a) } A=0 \text {; (b) } A=0.5, r>0
$$

Note, the parameter $r$ represents the horizontal direction of the attractors, if $r=0$, which means they perpendicular to the $X$-axis in horizontal direction, as shown in Figure 3(a); if $r<0$, which means the horizontal direction and $X$-axis at obtuse angle, as shown in Figure 3(b); and if $r>0$, which means the horizontal direction and X-axis at acute angle, as shown in Figure 2(b).

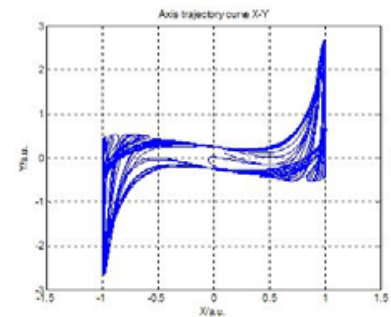

(a)

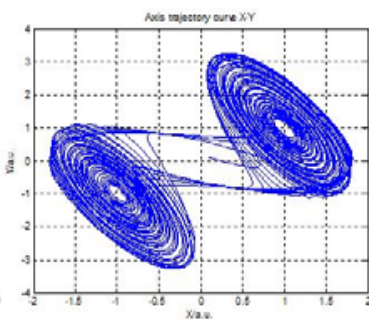

(b)
FIGURE III. DOUBLE SCROLL:(a) $r=0$; (b) $r<0$

1) Bifurcation: Case 1: $b=4.4, c=0.2, r=2.4$ and varying $a$, when $a \geq 1.35$, the bifurcation diagram of $x$ is given in Figure 4(a). Case 2: $a=4, c=0.2, r=2.4$ and varying $b$, when $b \epsilon[3.3$, 6.2], the bifurcation diagram of $x$ is given in Figure 4(b). Case 3: $a=4, b=4.4, r=2.4$ and varying $c$, when $c \epsilon(0.1,0.4)$ the bifurcation diagram of $x$ is given in Figure 4(c). We can see that the system shows abundant and sophisticated dynamical behaviors with parameters. 


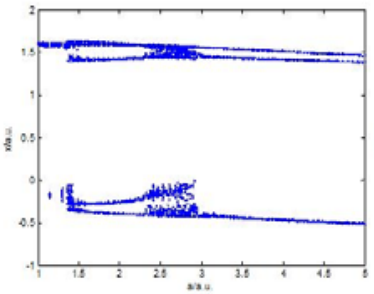

(a)

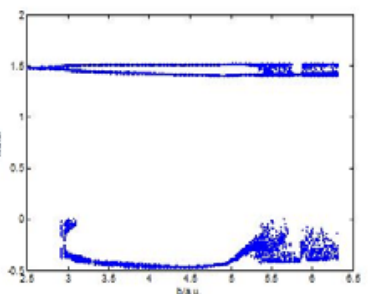

(b)

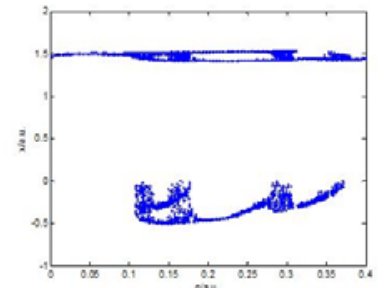

(c)

FIGURE IV. (a) BIFURCATION FOR $a$. (b). BIFURCATION FOR $b$. (c). BIFURCATION FOR $c$

2) Lyapunov exponent and Hausdroff dimension: The initial values is $(0.1,0.1,0.1)$. When $a=4, b=0.2, c=4.4, r=2.4$. The Lapunov exponent (LE) spectrum is shown in Figure 5.According to system (2) the LEs are as follows: $L E_{1}=4.02$, $L E_{2}=0, L E_{3}=-3.61$. Based on the Lyapunov exponents, we can calculate the Hausdroff dimension $D_{L}=2.102$.

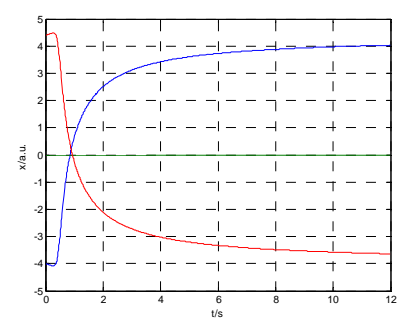

FIGURE V. LYAPUNOV EXPONENT

3) Poincaré maps: Poincaré section method is a direct and effective method which can observe and analyze complex dynamic behavior of system (2). It is depicted in Figure 6. It can be seen that it is consists of a series of isolated points, which means that the system is manifestly chaotic.

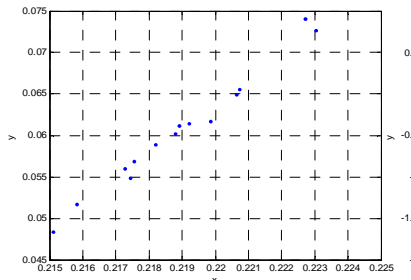

(a)

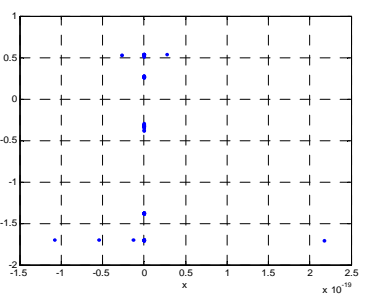

(b)
FIGURE VI. POINCARE MAPPING: (a) $z=0$; (b) $x+z=0$

\section{B. Higher Number of Scroll via Sign Function}

Define abbreviations and acronyms the first time they are used in the text, even after they have been defined in the abstract. Abbreviations such as IEEE, SI, MKS, CGS, SC, DC, and RMS do not have to be defined. Do not use abbreviations in the title or heads unless they are unavoidable.

To unified variables $(x, y, z)$, the dimensionless state equation of system (2) model is described by, let $a_{11}=-a, a_{12}=$ $r, a_{13}=0, a_{31}=-p, a_{32}=-q, a_{33}=-c$

$$
\left\{\begin{array}{l}
\frac{d x}{d t}=-a x+r y \\
\frac{d y}{d t}=b z \\
\frac{d z}{d t}=-p[x-f(x)]-q y-c z
\end{array}\right.
$$

where $x, y, z$ are state variables, and $a, b, c, r$ are real parameter. And $f(x)$ is the nonlinear sign function, which is the translational transformation criterion for constructing $2 N+1$ and $2(N+1)$-scroll, as below:

(1) $f_{1}(x)=f(x)$ is the first translational criterion, which can generate $N+1$-double scroll attractors as below:

$$
f_{1}(x)=\operatorname{Asgn}(x)+A \sum_{n=1}^{N}[\operatorname{sgn}(x+2 n A)+\operatorname{sgn}(x-2 n A)]
$$

When $A=0.5, a=0.4, p=q=b=r=1, c=0.6, N=0$ (NeR), the system can display double-scroll attractors, and $N=7$, the system can display 8-double scroll attractors, as shown in Figure 7.

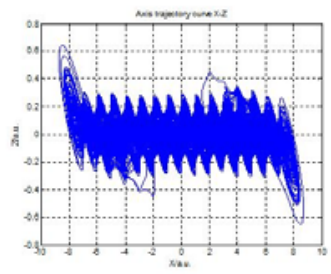

FIGURE VII. 8-DOUBLE SCROLL

(2) $f_{2}(x)=f(x)$ is the second translational criterion, which can generate $2 N+1$-scroll attractors as below:

$$
f_{2}(x)=A \sum_{n=1}^{N}\left\{\operatorname{sgn}[x+(2 n-1) A]+\sum_{n=1}^{N} \operatorname{sgn}[x-(2 n-1) A]\right\}
$$

When $A=1, a=0.3, p=q=1, b=0.4, r=2, c=0.6, N=1(N \epsilon R)$, the system can display 3 -scroll attractors, and $N=7$, the system can display 15- scroll attractors, as shown in Figure 8. 


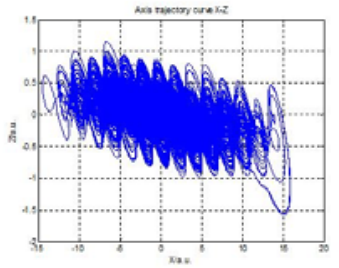

FIGURE VIII. 15- SCROLL

1) Equilibrium: $\operatorname{Real} A(A \geq 0)$ is related to the position of the equilibrium point, as follows: (1) In the chaotic system of $N+1$-double scroll attractors, at $( \pm 2 n A, 0,0)$; (2) In the chaotic system of $2 N+1$ - scroll attractors, at $( \pm(2 n-1) A, 0,0)$.

2) Bifurcation: Taking 2-double scroll attractors and $p=q=1$, as an example to investigate bifurcation. Case $1: r=2$, $b=0.4, c=0.6$ and varying $a$, when $a \epsilon[0,0.23]$, the bifurcation diagram of $x$ is given in Figure 9(a). Case 2: $a=0.2, b=0.4$, $c=0.6$ and varying $r$, when $r \epsilon[0.88,3)$, the bifurcation diagram of $x$ is given in Figure 9(b). Case 3: $a=0.2, r=2, c=0.6$ and varying $b$, when $b \epsilon(0,5]$ the bifurcation diagram of $x$ is given in Figure 9(c). Case 4: $a=0.2, r=2, b=0.4$ and varying $c$, when $c \epsilon(0,0.74]$ the bifurcation diagram of $x$ is given in Figure 9(c). We can see that the system shows abundant and sophisticated dynamical behaviors with parameters.

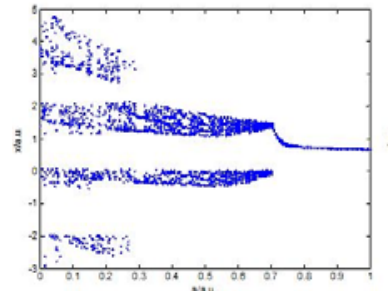

(a)

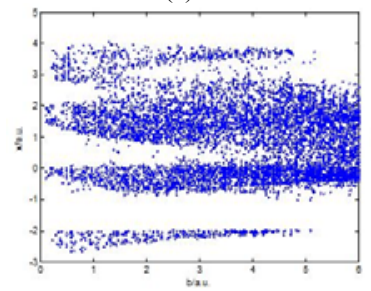

(c)

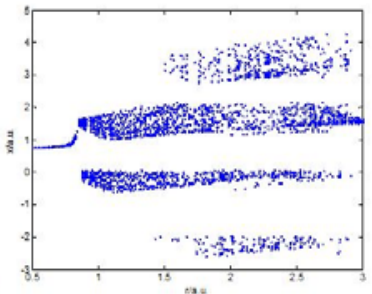

(b)

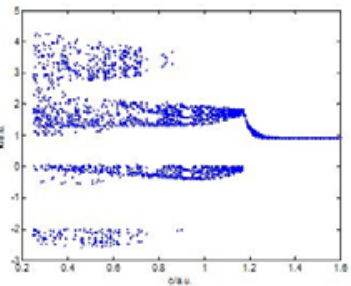

(d)
FIGURE IX. BIFURCATION (a) FOR $a$; (b) FOR $r$; (c) FOR $b$; (d) FOR $c$

3) Lyapunov exponent and Hausdroff dimension: The initial values is $(0.1,0.1,0.1)$. When $A=1, p=q=1, a=0.1, r=8$, $b=0.4, c=0.5,15$-scroll The Lapunov exponent (LE) spectrum is shown in Figure 10. According to system (5) the LEs are as follows: $L E_{1}=4.008, \quad L E_{2}=0, \quad L E_{3}=-3.93$. The Hausdroff dimension $\mathrm{DL}=2.02$.

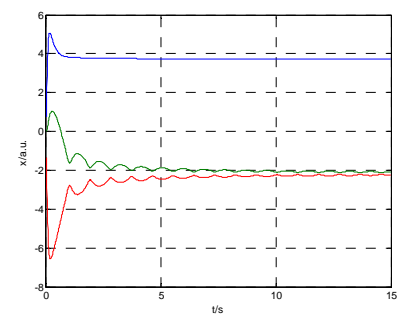

FIGURE X. LYAPUNOV MAPPING

4) Poincaré maps: The Poincaré mapping is depicted in Figure 11.

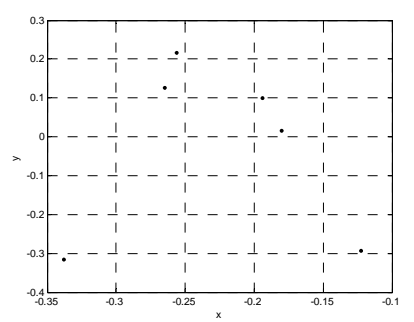

FIGURE XI. PONCARE EXPONENT

\section{Higher Number of Scrolls via Piecewise Functions}

Now, $f(x)$ is the nonlinear the piecewise function in the chaotic system (5), which is the translational transformation criterion for constructing $2 N+1$-and $2(N+1)$-scroll, as below:

(1) $f_{1}(x)=f(x)$ is the first translational criterion, which can generate $N+1$-double scroll attractors as below:

$$
\mathrm{f}_{1}(\mathrm{x})=\frac{\mathrm{A}}{\mathrm{m}} \sum_{\mathrm{n}=-\mathrm{N}}^{\mathrm{N}}[(x+2 n A)+m|-|(x+2 n A)-m \mid]
$$

When $A=1, \quad a=0.4, \quad p=q=1, \quad r=2, \quad b=1.2, \quad c=0.6$, $m=0.2$ (variable parameters), $N=0 \quad(N \epsilon R)$, the system can display double-scroll attractors, and $N=3$, the system can display 4-double scroll attractors, as shown in Figure 12.

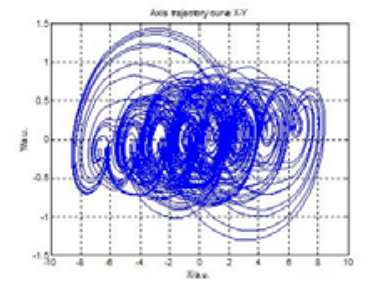

FIGURE XII. 7- DOUBLE SCROLL

(2) $f_{2}(x)=f(x)$ is the second translational criterion, which can generate $2 N+1$-scroll attractors as below:

$$
\mathrm{f}_{2}(\mathrm{x})=\frac{\mathrm{A}}{\mathrm{m}} \sum_{\substack{\mathrm{n}=\mathrm{N} \\ \mathrm{n} \neq 0}}^{\mathrm{N}}\left[\left|x+\left(2 n-\frac{|n|}{n}\right) A+m\right|-\left|x+\left(2 n-\frac{|n|}{n}\right) A-m\right|\right]
$$


When $A=1, a=0.1, \quad r=2, \quad p=q=1, \quad b=1.2, \quad c=0.6, \quad m=0.2$, $N=1(N \epsilon R)$, the system can display 3 -scroll attractors, and $N=3$, the system can display 7- scroll attractors, as shown in Figure 13 .

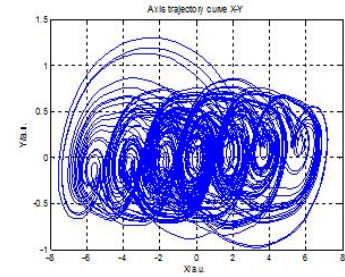

FIGURE XIII. 7- SCROLL

1) Equilibrium: Real $A(A \geq 0)$ and $m$ (variable parameters) are related to the position of the equilibrium point, as follows: (1) In the chaotic system of $N+1$-double scroll attractors, at $( \pm 2 n A, 0,0)$; (2) In the chaotic system of $2 N+1$ scroll attractors, at $( \pm[2 n-(|n| / n)] A, 0,0)$.

2) Lyapunov exponent and Hausdroff dimension: The initial values is $(0.1,0.1,0.1)$. When $A=1, p=q=1, a=0.1, r=2$, $b=1.2, c=0.6, m=0.2$, According to system (5) the LEs are as follows: $L E_{1}=0.04, \quad L E_{2}=0, \quad L E_{3}=-1.33$. The Hausdroff dimension $D_{L}=2.97$.

\section{Higher Number of Scrolls via Hyperbolic Function}

Now, $f(x)$ is the nonlinear the Hyperbolic function in the chaotic system (5), which is the translational transformation criterion for constructing $2 N+1$-and $2(N+1)$-scroll, as below:

(1) $f_{1}(x)=f(x)$ is the first translational criterion, which can generate $N+1$-double scroll attractors as below:

$$
\mathrm{f}_{1}(\mathrm{x})=\mathrm{A} \sum_{\mathrm{n}=-\mathrm{N}}^{\mathrm{N}} \tanh [m(x-2 n A])
$$

When $A=1, \quad a=0.3, \quad r=2, \quad p=q=1, \quad b=1.5, \quad c=0.6, m=6$ (variable parameters), $N=0 \quad(N \epsilon R)$, the system can display double-scroll attractors, and $N=4$, the system can display 5double scroll attractors, as shown in Figure 14.

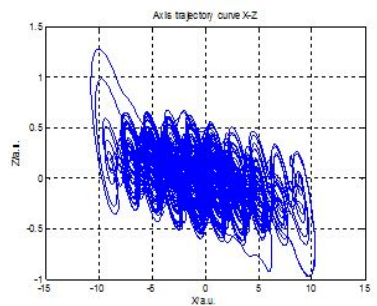

FIGURE XIV. 8-DOUBLE SCROLL

(2) $f_{2}(x)=f(x)$ is the second translational criterion, which can generate $2 N+1$-scroll attractors as below:

$$
\mathrm{f}_{2}(\mathrm{x})=\mathrm{A} \sum_{\substack{\mathrm{n}=\mathrm{N} \\ \mathrm{n} \neq 0}}^{\mathrm{N}} \tanh \left\{m\left[x-\left(2 n-\frac{|n|}{n}\right) A\right]\right\}
$$

When $A=1, a=0.3, p=q=1, \quad r=1.1, \quad b=1.2, \quad c=0.6, \quad m=6$, $N=0(N \epsilon R)$, the system can display single scroll attractors, and $N=4$, the system can display 9- scroll attractors, as shown in Figure 15.

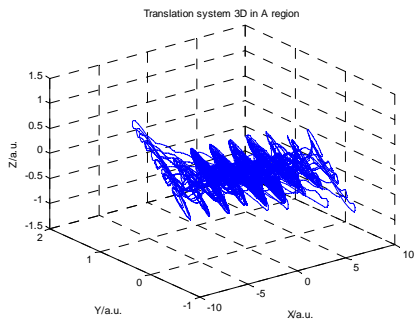

FIGURE XV.9- SCROLL

1) Equilibrium: Real $A(A \geq 0)$ and $m$ (variable parameters) are related to the position of the equilibrium point, as follows: (1) In the chaotic system of $N+1$-double scroll attractors, at $( \pm 2 n A, 0,0)$; (2) In the chaotic system of $2 N+1$ scroll attractors, at $( \pm[2 n-(|n| / n)] A, 0,0)$.

2) Lyapunov exponent and Hausdroff dimension: The initial values is $(0.1,0.1,0.1)$. When $A=1, a=0.1, p=q=1, r=7$, $b=1.2, c=0.5, m=5.5$, According to system (5) the LEs are as follows: $L E_{1}=2.741, L E_{2}=0, L E_{3}=-2.365$. The Hausdroff dimension $D_{L}=2.137$.

\section{CONCLUSION}

In this paper, generation $N$-scroll existence four different functions are been proposed of the translation chaotic system. They are absolute function, sign function, the piecewise and hyperbolic function. Then, the dynamics properties are investigated in detail. Furthermore, MATLAB simulation results show that these functions emerge similar but topologically non-equivalent chaotic attractors. Undoubtedly these results will improve this chaotic system application value in the future.

\section{REFERENCES}

[1] T Hikihara, P Holmes, T Kambe, and et al,"Introduction to the focus issue: Fifty years of chaos: Applied and theoretical", Chaos, vol 22, 2012, pp. 047501 .

[2] W. L Ditto, and K Showalter,"Introduction: Control and synchronization of chaos",Chaos,vol 7, 1997, pp. 509-511.

[3] C Stegemann, H. A Albuquerque, R.M Rubinger, and et al, "Lyapunov exponent diagrams of a 4-dimensional Chua system", Chaos, vol 21, 2011, pp. 033105.

[4] J. G Zhang, Y. D Chu, W. J Du, and et al, "Hopf bifurcation analysis in a new chaotic system with chaos entanglement function", J. of Appl. Math. 2014, pp. 371509.

[5] Y. I Boev, T. E Vadivasova, and V. S Anishchenko,"Poincaré recurrence statistics as an indicator of chaos synchronization", Chaos, vol 24, 2014, pp. 023110.

[6] W. Jin, and F Chen, "Topological chaos of universal elementary cellular automata rule", Nonlinear Dyn, vol 63, 2011,pp. 217-222.

[7] E. R Viana, R. M Rubinger, H. A Albuquerque, and et al, "Periodicity detection on the parameter-space of a forced Chua's circuit", Nonlinear Dyn, vol 67(1), 2012, pp. 385-392.

[8] I Stewart, "The Lorenz attractor exists", Nature, vol 37(4), 2000, pp. 341-347 
[9] N. J Corron, J. N Blakely, and M. T Stahl, "A matched filter for chaos", Chaos, vol 20, 2010, pp. 023123.

[10] T. He, and S. Habib, “Chaos and noise”, Chaos, vol 23, 2013, pp. 033123

[11] B Kia, S Kia, J. F Lindner, and et al, "Noise tolerant spatiotemporal chaos computing", Chaos, vol 24, 2014, pp. 043110(2014).

[12] D. Younesian, and H. Norouzi, "Chaos prediction in nonlinear viscoelastic plates subjected to subsonic flow and external load using extended Melnikov's method", Nonlinear Dyn, vol 84 (3), 2016, pp. 1163-1179.

[13] L. Minati,“ Experimental synchronization of chaos in a large ring of mutually coupled single-transistor oscillators: Phase, amplitude, and clustering effects", Chaos, vol 24, 2014, pp. 043108.

[14] Y Hirata, M Oku, and K Aihara, "Chaos in neurons and its application: Perspective of chaos engineering", Chaos, vol 22, 2012, pp. 047511.

[15] Z.X.Yuan, and G.H. Huang, "Chaos-based encryption for fractal image coding", Chin. Phys. B, vol 21(1), 2012, pp. 115-126.

[16] J Pan, Q Ding, and B.X Du,"A new improved scheme of chaotic masking secure communication based on Lorenz system", Int. J. Bifurcation and Chaos, vol 26(4), 2016, pp. 1650072

[17] N. D Caranicolas, and E. E Zotos,"The evolution of chaos in active galaxy models with an oblate or a prolate dark halo component", Astronomische Nachrichten, vol 331(3), 2010, pp. 330-337.

[18] S Čelikovský, and G Chen, “On a generalized Lorenz canonical form of chaotic systems", Int. J. Bifurcation and Chaos, vol 12(8), 2002, pp. 1789-1812

[19] T. S Zhou, G. R Chen, and S Čelikovský, "Ši'linikov chaos in the generalized Lorenz canonical form of dynamical systems", Nonlinear Dyn, vol 39, 2005, pp. 319-334.

[20] C Zhang, S Yu, J Lü, and et al, "Generating multi-wing butterfly attractors from the piecewise-linear Chen system", 2008 9th International Conference for Young Computer Scientist, vol 18-21, 2008, pp. 2840-2845

[21] Q.H Alsafasfeh, and M.S Al-Arni,“A new chaotic behavior from Lorenz and Rössler systems and its electronic circuit implementation”, Circuits and Systems, vol 2, 2011, pp. 101-105.

[22] L.O Chua, M Komuro, and T Matsumoto,"The double scroll family", IEEE Trans. Circ. Syst.-I : Fund. Th. Appl, vol 33(11), 1986, pp. 10731118

[23] J.A.K. Suykens, and J. Vandewalle,"Generation of N-double scrolls $(\mathrm{n}=1,2,3,4, \ldots)$ ", IEEE Trans. Circ. Syst.-I : Fund. Th. Appl, vol 40(11), 1993, pp. 861-867

[24] S Yu, J Lü, and G Chen,"Multi folded torus chaotic attractors: Design and implementation", Chaos, vol 17,2007, pp. 013118

[25] W.K.S Tang, G.Q Zhong, and K.F Man, "Generation of $N$-scroll attractors via sine function", IEEE Transactions on Circuits \& Systems-I: Fundamental Theory and Applications, vol 48(11), 2001, pp. 1369-1372.

[26] J Lü,"Generating multi-scroll chaotic attracters: Theories, methods and applications", International Journal of Bifurcation and Chaos, vol 16(4), 2006, pp. 775-858.

[27] C Zhang, and S Yu, "Generation of grid multi-scroll chaotic attractors via switching piecewise linear controll”, Phys. Lett. A, vol 374(30), 2010, pp. 29-37.

[28] X Ai, K Sun, S He, and et al, "Design of grid multi-scroll chaotic attractors via transformations", Int. J. Bifurcation and Chaos, vol 25(10), 2015, pp. 1530027

[29] Y Liu, J Guan, C.M Yang, and et al,"Generation of $2 N+1$-scroll existence in new three-dimensional chaos systems", Chaos, vol 26, 2016, pp. 084307.

[30] Y Liu, and S Guo," Generation and dynamics analysis of N-scrolls existence in new translation-type chaotic systems", Chaos, vol 26 (11), 2016, pp. 113114. 